\title{
DA NECESSIDADE DE SE ADOTAR E DIVULGAR ESQUEMAS TERAPÊUTICOS PARA TRATAMENTO DE LEISHMANIOSE TEGUMENTAR NO PARANÁ
}

\author{
Ueslei TEODORO (1), Roberto PaIma SPINOZA (2), Vicente LA SALVIA FILHO (1), Ana Lúcia Falavigna GUILHERME \\ (1), Airton Pereira LIMA (3), Gabriel Marcelo Botelho JUNQUEIRA (3), Norico Miyagui MISUTA (3), Antonio NERILO \\ SOBRINHO (1) \& Edson Mauricio de LIMA (1)
}

\begin{abstract}
RESUMO
A leishmaniose tegumentar tem mostrado caráter endêmico no Norte-Noroeste do Paraná, em áreas que vêm sendo ocupadas desde os anos 40 , onde persistem matas residuais com modificações bastante acentuadas. A infecção tem atingido sobretudo adultos do sexo masculino, mas também vem sendo constatada em mulheres e crianças, e por vezes em diversos componentes de uma mesma família. Investigou-se 513 casos de leishmaniose tegumentar notificados pela SUCAM, com informações de terem tido apenas diagnóstico clínico, de janeiro de 1987 a agosto de 1989, os quais iniciaram o tratamento com Glucantime ${ }^{\circledR}$, em Centros de Saúde do Estado ou na própria SUCAM. De 513 pacientes tratados $260(50,67 \%)$ tiveram alta por cura clínica e os $253(49,33 \%)$ restantes não retornaram para verificação da cura clínica ou abandonaram o tratamento. A quantidade de ampolas de Glucantime ${ }^{\circledR}$ fornecidas para os pacientes oscilou de 10 a mais de 200 , com ou sem intervalo durante o tratamento, sendo que $217(85,46 \%)$ dos 260 casos receberam de 10 a 75 ampolas. A ocorrência de leishmaniose em $163(31,75 \%)$ mulheres e em $34(6,62 \%)$ crianças, de ambos os sexos, até dez anos de idade, sugere a transmissão domiciliar. Não se sabe se os $253(49,33 \%)$ indivíduos que não retornaram ou abandonaram o tratamento seguiram a prescrição terapêutica e se restabeleceram. $\mathrm{O}$ elevado número de indivíduos que não retornou ou abandonou o tratamento mostra a necessidade de maior conscientização das consequências que podem advir do tratamento incompleto. APOIO: Núcleo de Estudos em Saúde Coletiva.
\end{abstract}

UNITERMOS: Leishmaniose tegumentar americana; Tratamento; Glucantime ${ }^{\circledR}$

\section{INTRODUÇĀO}

Nas Américas Central e do Sul as leishmanioses tegumentares têm sido descritas em quase todos os países, verificando-se que a distribuição das diversas formas clínico-epidemológicas guarda estreita relação com as condições florísticas e faunísticas ${ }^{22}$. No Brasil continuam sendo apontadas como doenças importantes do ponto de vista da saúde pública, com ampla distribuição por quase todo o território nacional ${ }^{4,7,13,15}$.

O risco de infecção, classicamente atribuído às formas de ocupação dos ambientes florestais pelo homem, ressurge agora com outra aparência em áreas onde focos ativos da doença sobreviveram em matas residuais ${ }^{1,3,8}$. O reaparecimento de casos humanos nessas áreas, após longo período de quiescência, reflete a carência de investigações que poderiam trazer melhorias para as condutas clássicas de controle e tratamento ${ }^{22}$. Acrescente-se que a subnotificação e a falta de dados completos na notificação têm retratado de maneira irreal a incidência da doença ${ }^{22}$

(1) Fundação Universidade Estadual de Maringá, Paraná, Brasil.

(2) Superintendência de Campanhas de Saúde Pública (SUCAM), Londrina, Paraná, Brasil

(3) Secretaria de Estado de Saúde, Maringá, Paraná, Brasil.

Endereço para correspondência: Dr. Ueslei Teodoro. Departamento de Análises Clínicas, Fundação Universidade Estadual de Maringá. Av. Colombo, 3690, Telefone 0442 26-2727 ramal 347 CEP 87020, Maringá, Paraná, Brasil. 
TEODORO, U., SPINOZA, R.P.; LA SALVIA FILHO, V.; GUILHERME, A.L.F.; LIMA, A.P.; JUNQUEIRA, G.M.B.; MISUTA,

N.M.; NERILO SOBRINHO, A. \& LIMA, E.M. de - Da necessidade de se adotar e divulgar esquemas terapêuticos para tratamento de leishmaniose tegumentar no Paraná. Rev. Inst. Med. trop. S. Paulo, 33 (3): 199-204, 1991.

A leishmaniose vem mostrando caráter endêmico nas regiões Norte-Noroeste do Paraná desde 1980, ano em que foi pela primeira vez notificada oficialmente pela SUCAM ${ }^{19}$, embora conste desde 1975, nos arquivos de um laboratório de patologia da cidade de Maringá, o registro de vários casos da doença ${ }^{24}$. Além disso, a própria SUCAM (Distrito de Londrina) registrou 67 casos de leishmaniose nos municípios de Terra Boa e Jussara, em $1978^{18}$. Endêmica nessas regiões esta zoonose indica mudanças na dinâmica de transmissão do tipo silvático para o tipo persistente em áreas que sofreram intensa transformação ambiental ${ }^{5}$. Nessas regiões foi isolado e classificado como agente etiológico Leishmania (Viannia) braziliensis $^{24}$, e mais recentemente Leishmania (Leishmania) amazonensis, de paciente que se infectou no minicípio de Floresta - Paraná ${ }^{17}$.

A terapia da leishmaniose tegumentar, especialmente leishmaniose cutâneo-mucosa, às vezes, é problemática e deveria exigir maior atenção dos serviços de saúde. Mediante esta situação os participantes da Reunião Técnico Operacional Sobre o Controle da Leishmaniose Tegumentar Americana (LTA), no Brasil ${ }^{13}$ elaboraram um relatório onde indicam esquemas terapêuticos para a leishmaniose cutânea e/ou mucocutânea. As dificuldades citadas por WARD ${ }^{25}$, LAINSON ${ }^{8}$, MARSDEN ${ }^{11,12}$, TALHARI ${ }^{20}$, TALHARI et al. ${ }^{21}$ e SAMPAIO et al ${ }^{16}$, referentes ao diagnóstico, aquisição e custo do antimoniato de $\mathrm{N}$-metil glucamina (Glucantime ${ }^{\circledR}$ ), ao tratamento prolongado com uma ou mais séries de injeções de Glucantime ${ }^{\circledR}$, aplicações com ou sem intervalo de tempo, abandono de tratamento e, finalmente, a contração da infecção por trabalhadores em áreas distantes, onde as comunicações e assistência médica são frequentemente inadequadas, são situações vivenciadas no Paraná apesar da existência de razoável infra-estrutura médico-assistencial.

O principal objetivo deste trabalho foi expor a problemática do tratamento da leishmaniose tegumentar no Norte-Noroeste do Paraná e, paralelamente, tentou-se traçar o perfil da endemia nestas regiões.

\section{MATERIAL E MÉTODOS}

Área estudada. As regiões Norte-Noroeste do Paraná vem sendo ocupadas desde os anos 40, quando iniciou-se grande desmatamento nestas regiões. A vegetação nativa, já em grande parte destruída, é formada por mata densa do tipo tropical de transição para subtropical. Das florestas nativas restam poucas e esparsas manchas de vegetação, geralmente modificadas. a paisagem no seu conjunto é de planalto, com clima tropical de transição para subtropical, temperaturas médias anuais entre 20 e $22^{\circ} \mathrm{C}$ e média de mês mais quente superior a $22^{\circ} \mathrm{C}$. A precipitação anual avaliada por um período de 5 anos, no município de Cianorte, indicou média de $1600 \mathrm{~mm} / \mathrm{ano}$.

Coleta de dados. As informações sobre os pacientes com diagnóstico clínico e tratados com Glucantime ${ }^{\circledR}$ foram colhidas de formulários de controle de tratamento usados pela SUCAM - Distrito de Londrina, Pr., no período de janeiro de 1987 a agosto de 1988. As informações abrangeram idade, sexo, procedência, casos familiares, casos com lesão nasal, bucal ou bucofaríngea, número de ampolas de Glucantime ${ }^{\circledR}$ por paciente e a evolução do tratamento com avaliação clínica. Estes dados foram confrontados entre si originando as tabelas presentes neste trabalho.

\section{RESULTADOS}

A idade dos pacientes variou de 2 meses a 78 anos. Nota-se na tabela 1 que a infecção ocorreu em pessoas de diversas faixas etárias mas sobretudo naquelas de 11 a 50 anos (76,23\%). A mesma tabela mostra que houve predominância de infecção nos indivíduos do sexo masculino $(68,25 \%)$. A taxa de infecção $(31,75 \%)$ no sexo feminino foi elevada e 34 crianças de ambos os sexos, na faixa de 0 a 10 anos também se infectaram, ressaltando-se que 12 desas crianças eram de famílias com adultos infectados (Tabela 1). O número de ampolas de Glucantime ${ }^{\circledR}$ variou de 10 a mais de 200 por pacientes, destacando-se que de 513 pacientes $260(50,67 \%)$ obtiveram alta por cura clínica e os demais abandonaram o tratamento ou não retornaram para avaliação de cura (Tabela 2). Dos 260 que tiveram alta $217(85,46 \%)$ foram medicados com uma quantidade de Glucantime ${ }^{\circledR}$ que oscilou de 10 a 75 ampolas (Tabela 3). Constatou-se que 395 (77\%) pacientes eram autóctones do Estado do Paraná, distribuídos em 69 municípios, sobretudo do Norte-Noroeste do Paraná, sendo $157(39,65 \%)$ dos municípios de Jussara, Cianorte e Terra Boa; 91 casos procediam de outros Estados e de 27 não foi possível determinar a origem (Tabela 4; Figura 1). Foram notificados 44 casos com lesão nas mucosas nasal e/ou bucofaríngea, dos quais apenas 11 obtiveram alta por cura clínica. 
TEODORO, U., SPINOZA, R.P.; LA SALVIA FILHO, V.; GUILHERME, A.L.F.; LIMA, A.P.; JUNQUEIRA, G.M.B.; MISUTA,

N.M.; NERILO SOBRINHO, A. \& LIMA, E.M. de - Da necessidade de se adotar e divulgar esquemas terapêticos para tratamento de leishmaniose tegumentar no Paraná. Rev. Inst. Med. trop. S. Paulo, 33 (3): 199-204, 1991.

TABELA 1 - Distribuição de pacientes tratados com glucantime segundo a faixa etária e sexo.

\begin{tabular}{|c|c|c|c|c|c|c|}
\hline \multirow{2}{*}{$\begin{array}{l}\text { Faixa } \\
\text { Etária }\end{array}$} & \multicolumn{4}{|c|}{ Sexo } & \multicolumn{2}{|c|}{ Total } \\
\hline & $\overline{\mathrm{M}}$ & $\%$ & $F$ & $\%$ & & $\%$ \\
\hline $\begin{array}{lll}0 & \text { a } 10\end{array}$ & 12 & 2,34 & 22 & 4,28 & 34 & 6,62 \\
\hline 11 a 20 & 62 & 12,11 & 33 & 6,43 & 95 & 18,54 \\
\hline 21 a 30 & 88 & 17,15 & 31 & 6,04 & 119 & 23,19 \\
\hline 31 a 40 & 74 & 14,42 & 29 & 5,65 & 103 & 20,07 \\
\hline 41 a 50 & 54 & 10,53 & 20 & 3,90 & 74 & 14,43 \\
\hline 51 a 60 & 34 & 6,63 & 12 & 2,33 & 46 & 8,96 \\
\hline 61 ou + & 21 & 4,10 & 16 & 3,12 & 37 & 7,22 \\
\hline ñ consta & 5 & 0,97 & 0 & 0,00 & 5 & 0,97 \\
\hline Total & 350 & 68,25 & 163 & 31,75 & 513 & 100,00 \\
\hline
\end{tabular}

TABELA 2 - Quantidade de glucantime utilizado por pacientes no tratamento de leishmaniose tegumentar no Paraná.

\begin{tabular}{|c|c|c|c|c|c|c|}
\hline \multirow{2}{*}{$\begin{array}{l}\text { № De } \\
\text { Ampolas de } \\
\text { Glucantime }\end{array}$} & \multicolumn{2}{|c|}{$\begin{array}{l}\text { Pacientes com } \\
\text { Cura clínica }\end{array}$} & \multicolumn{2}{|c|}{$\begin{array}{l}\text { Pacientes que } \\
\text { Não Retornaram }\end{array}$} & \multicolumn{2}{|c|}{$\begin{array}{l}N^{\circ} \text { de } \\
\text { Pacientes }\end{array}$} \\
\hline & № & $\%$ & $\mathrm{~N}^{\mathrm{o}}$ & $\%$ & TOTAL & $\%$ \\
\hline 10 a 25 & 81 & 15,79 & 37 & 7,21 & 118 & 23,0 \\
\hline 26 a 50 & 93 & 18,13 & 109 & 21,25 & 202 & 39,38 \\
\hline 51 a 75 & 43 & 8,38 & 55 & 10,72 & 98 & 19,10 \\
\hline 76 a 100 & 15 & 2,92 & 33 & 6,44 & 48 & 9,36 \\
\hline 101 a 150 & 15 & 2,92 & 15 & 2,93 & 30 & 5,85 \\
\hline 151 a 200 & 11 & 2,14 & 4 & 0,78 & 15 & 2,92 \\
\hline+ de 200 & 2 & 0,39 & 0 & 0,00 & 2 & 0,39 \\
\hline Total & 260 & 50,67 & 253 & 49,33 & 513 & 100,00 \\
\hline
\end{tabular}

TABELA 3 - Quantidade de glucantime por pacientes que obtiveram alta por cura clínica.

\begin{tabular}{ccc}
\hline $\begin{array}{l}\text { No de Ampolas } \\
\text { de Glucantime }\end{array}$ & \multicolumn{2}{c}{ Pacientes com } \\
& $N^{\circ}$ & $\%$ \\
\hline 10 a 25 & 81 & 31,15 \\
26 a 50 & 93 & 37,77 \\
51 a 75 & 43 & 16,54 \\
76 a 100 & 15 & 5,77 \\
101 a 150 & 15 & 5,77 \\
151 a 200 & 11 & 4,23 \\
+ de 200 & 2 & 0,77 \\
\hline TOTAL & 260 & 100,00 \\
\hline
\end{tabular}

\section{DISCUSSÃO}

O predomíneo de leishmaniose tegumentar no sexo masculino no Norte-Noroeste do Paraná pode ser parcialmente explicado pela maior exposição dos homens em atividades de lazer (caça e pesca) e mesmo durante o trabalho, como foi observado anteriormente por VERZIGNASSI et al. ${ }^{24}$. Por outro lado os casos familiares com a presença de numerosas mulheres e crianças sugerem fortemente a transmissão domiciliar, o que ocorre em função de
TABELA 4 - Distribuição dos casos de leishmaniose tegumentar em 69 municípios do Paraná.

\begin{tabular}{|c|c|c|c|}
\hline Município & $\begin{array}{l}N^{\circ} \mathrm{de} \\
\text { Casos }\end{array}$ & Município & $\begin{array}{l}N^{\circ} \text { de } \\
\text { Casos }\end{array}$ \\
\hline 01 - Arapongas & 2 & 36-Jataizinho & 4 \\
\hline 02 - Araruna & 3 & 37 - Jussara & 94 \\
\hline 03 - Assis Chateaubreand & 1 & 38-Kaloré & 4 \\
\hline 04 - Atalaia & 1 & 39- Lobato & 7 \\
\hline 05- Barbosa Ferraz & 2 & 40- Londrina & 11 \\
\hline 06- Cambira & 4 & 41- Mamborê & 2 \\
\hline 07- Campo Mourão & 4 & 42- Mandaguari & 2 \\
\hline 08- Candido de Abreu & 1 & 43- Marilândia do Sul & 5 \\
\hline 09- Centenário do Sul & 1 & 44- Maringá & 4 \\
\hline 10- Cianorte & 38 & 45- Marumbi & 1 \\
\hline 11- Cidade Gaúcha & 1 & 46- Mirasselva & 1 \\
\hline 12- Colombo & 1 & 47- Moreira Sales & 1 \\
\hline 13-Colorado & 15 & 48- Munhoz de Melo & 2 \\
\hline 14- Cruzeiro do Sul & 5 & 49- Nova Esperança & 3 \\
\hline 15- Diamante do Norte & 1 & 50- Ortigueira & 1 \\
\hline 16- Dr. Camargo & 13 & 51- Ourizona & 3 \\
\hline 17- Engenheiro Beltrão & 17 & 52- Paraíso do Norte & 1 \\
\hline 18- Faxinal & 1 & 53- Paranacity & 5 \\
\hline 19- Fênix & 1 & 54- Paranapoema & 1 \\
\hline 20-Floraí & 1 & $55-1^{9}$ de Maio & 7 \\
\hline 21- Floresta & 3 & 56- Querência do Norte & 2 \\
\hline 22- Florestópolis & 1 & 57- Quinta do Sol & 4 \\
\hline 23- Flórida & 1 & 58- Rondon & 20 \\
\hline 24- Goio Erê & 6 & 59- Santa Fé & 3 \\
\hline 25- Ibiporã & 2 & 60- Santo Inácio & 1 \\
\hline 26- Inajá & 1 & 61- São Jerônimo da Serra & 1 \\
\hline 27-Iretama & 1 & 62- São Jorge do Ivaí & 11 \\
\hline 28-Itaguajé & I & 63- São Pedro do Ivaí & 1 \\
\hline 29- Ivaiporã & 3 & 64-São Tomé & 8 \\
\hline 30- Jaguapitã & 1 & 65-Sertanópolis & 2 \\
\hline 31- Jandaia do Sul & 1 & 66- Тегта Воa & 25 \\
\hline 32- Janiópolis & 2 & 67- Ubiratã & I \\
\hline 33- Japurá & 6 & 68- Umuarama & I \\
\hline 34- Jardim Alegre & 11 & 69- Uraí & 1 \\
\hline \multirow[t]{2}{*}{ 35- Jardim Olinda } & 2 & 70- Importado & 91 \\
\hline & 4 & 71 - Indeterminado & 27 \\
\hline
\end{tabular}

transformações no ambiente dos flebotomíneos, os quais por sua vez passam a invadir o domicílio e o peridomicílio humano, como vem sendo observado por diversos investigadores nos Estados de São Paulo ${ }^{3,6}$, Rio de Janeiro ${ }^{9,14}$, Espírito Santo ${ }^{1}$, Minas Gerais ${ }^{6,10}$ e Paraná ${ }^{2}$.

Em 1986, após entendimentos entre a Universidade Estadual de Maringá, SUCAM e Secretaria de Estado de Saúde, através dos Distritos Sanitários de Maringá e Cianorte, concluiu-se que era preciso divulgar informações à população e aos serviços de assistência médica sobre a frequência e a situação grave da doença no Norte-Noroeste do Paraná. Até 1986, conforme testemunha de alguns autores desse trabalho, em diversas localidades da região em apreço eram frequentes o diagnóstico e tratamento errôneos, além de que os portadores da 
TEODORO, U., SPINOZA, R.P.; LA SALVIA FILHO, V.; GUILHERME, A.L.F.; LIMA, A.P.; JUNQUEIRA, G.M.B.; MISUTA, N.M.; NERILO SOBRINHO, A. \& LIMA, E.M. de - Da necessidade de se adotar e divulgar esquemas terapêuticos para tratamento de leishmaniose tegumentar no Paraná. Rev. Inst. Med. trop. S. Paulo, 33 (3): 199-204, 1991.

Figura 1 - Municípios do Estado do Paraná com notificações de leishmaniose tegumentar americana.

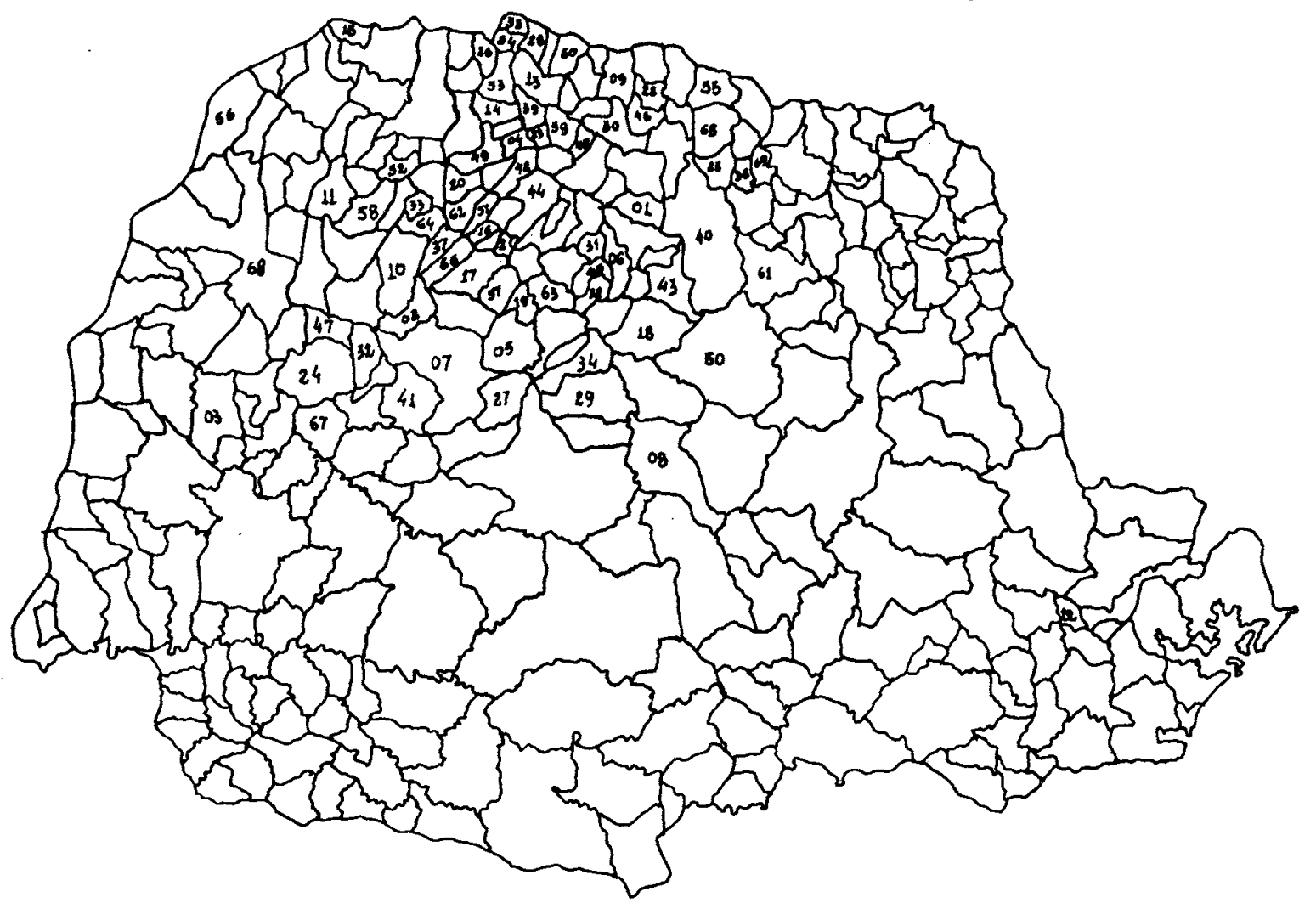

Os números desta figura correspondem à mesma sequência dos municípios apresentados na Tabela 4.

infecção procuravam primeiramente as farmácias onde eram medicamentados usualmente com penicilina (Benzetacil@). Ressalta-se ainda que por diversas vezes o tratamento dos doentes era adiado ou interrompido pela inexistência de Glucantime ${ }^{\circledR}$ nos órgãos responsáveis pelo tratamento dos doentes, e inclusive em farmácias comerciais. Atualmente a Secretaria de Saúde de Estado vem suprindo toda a demanda de Glucantime ${ }^{\circledR}$. Desde então os postos de atendimento médico da Secretaria de Saúde de Estado e dos Municípios, principalmente de Maringá e Cianorte, passaram a ter maior preocupação com a problemática do diagnóstico e tratamento, o que vem resultando em melhor atendimento aos pacientes com infecção. $O$ fato de 260 $(50,67 \%)$ indivíduos terem obtido cura clínica demonstrou que houve maior acesso ao diagnóstico e tratamento da leishmaniose, tendo em vista que apenas $35,6 \%$ dos pacientes tiveram alta por cura clínica, nos anos de 1987 e $1988^{23}$. Cabe lembrar que as dificuldades supramencionadas pára o diagnóstico e tratamento de leishmaniose, ocorrem também no Norte-Noroeste do Paraná $8,11,12,16,20,21,25$.
Portanto não se pode perder de vista que a existência de $253(49,23 \%)$ pacientes que abandonaram o tratamento, ou não retornaram para verificação de cura, mostra ser preciso dar continuidade ao processo de divulgação de informações no Paraná sobre as consequências mais graves que podem advir da parasitose, na ausência de tratamento ou tratamento inadequado.

Os Pacientes que receberam alta foram medicados com quantidade variável de Glucantime ${ }^{\circledR}$, seguindo-se esquema terapêutico de acordo com o critério adotado pelos médicos envolvidos no tratamento, existindo ou não intervalo de tempo entre as doses do medicamento. Observou-se que o tratamento tardio e/ou incompleto dificultava a cura e requeria maior quantidade de Glucantime ${ }^{\circledR}$, confirmando a hipótese de que o diagnóstico precoce e o tratamento imediato reduzem a dose da droga e evitam o desconforto de uma doença prolongada ${ }^{8}$.

Dos casos autóctones do Estado do Paraná, 
TEODORO, U., SPINOZA, R.P.; LA SALVIA FILHO, V.; GUILHERME, A.L.F.; LIMA, A.P.; JUNQUEIRA, G.M.B.; MISUTA, N.M.; NERILO SOBRINHO, A. \& LIMA, E.M. de - Da necessidade de se adotar e divulgar esquemas terapêuticos para tratamento de leishmaniose tegumentar no Paraná. Rev. Inst. Med. trop. S. Paulo, 33 (3): 199-204, 1991.

40\% eram dos Municípios de Jussara, Cianorte e Terra Boa, que fazem divisa entre si e situam-se no vale do rio Ivaí, constituindo área de alto risco de infecção pela grande concentração de matas residuais nesses municípios.

A persistência de casos com envolvimento nasobucofaríngeo e o elevado número desses pacientes que não retornaram para verificação de cura clínica, ou abandonaram o tratamento, na região Norte-Noroeste do Paraná, podem estar também relacionados às dificuldades acima comentadas $8.11,12.16,20,21,25$. Isso demonstra uma vez mais a necessidade de se aperfeiçoar os serviços de atendimento à leishmaniose tegumentar no Paraná.

\section{RECOMENDAÇŌES}

1- deve-se procurar adotar e divulgar melhor os esquemas terapêuticos indicados no RelatórioOperacional sobre Controle de Leishmaniose Tegumentar Americana no Brasil;

2- o elevado número de indivíduos que não retornou ou abandonou o tratamento mostra a necessidade de maior esclarecimento das consequências que podem advir do tratamento incompleto ou da inexistência deste;

3- deve-se procurar sensibilizar o pessoal envolvido nos serviços de assistência médica e os pacientes da necessidade do acompanhamento médico no decorrer do tratamento, para verificação de cura clínica e para que se evite com maior segurança a evolução para formas mutilantes, o que ainda assim não descarta a possibilidade de recidivas;

4- é imprescindível que se crie condições para a realização de diagnóstico laboratorial no estado do Paraná; no mínimo o esfregaço por aposição e a intradermorreação de Montenegro;

5- não se sabe se os indivíduos que não retornam ou abandonaram tratamento seguiram a prescrição terapêutica e se restabeleceram;

6- que se mantenha um estoque estratégico de Glucantime ${ }^{\circledR}$ nos órgãos responsáveis pelo tratamento dos doentes;

7- que seja aprimorada a coleta de informações dos pacientes nos serviços de notificação;
8- a ocorrência de casos familiares, especialmente em mulheres e crianças, de ambos os sexos, sugere a transmissão domiciliar;

9- é necessário estimular estudos sobre reservatórios e vetores para que se tenha maior compreensão da epidemiologia da leishmaniose tegumentar nas regiões Norte-Noroeste do Paraná.

\section{SUMMARY}

\section{It's necessary to adopt and disseminate therapeutic schemes for the treatment of cutaneous leishmaniasis in Paraná State.}

American cutaneous leishmaniasis has shown an endemic character in areas of North-North-west Paraná State, which were populated in 40's, where residual forests persist with intense modifications. The infection has been found mainly in males, but was found also in females and children and in many individuals of a same family. 513 cases of cutaneous leishmaniasis notified to SUCAM by clinical diagnosis were investigated. Treatment was begun with Glucantime ${ }^{\circledR}$ at State Health Centers or at SUCAM. Of 513 treated patients 260 $(50.67 \%)$ were considered clinically cured. The remaining $253(49.33 \%)$ abandoned treatment. The quantity of Glucantime ${ }^{\circledR}$ supplied to the patients varied from 10 vials to more than 200 , with or without intervals during the treatment. Two hundred and seventeen $(85.46 \%)$ patients among 260 received from 10 to 75 vials. The ocurrence of leishmaniasis in $163(31.75 \%)$ women and in 34 $(6.62 \%)$ children, of both sexes, up to 10 years old, suggests domiciliary transmission. It's not known if the $253(49.33 \%)$ individuals who abandoned the treatment recovered. This high number shows the necessity of better knowledge of the consequences that can occur due to incomplete treatment.

\section{REFERÊNCIAS BILBIOGRÁFICAS}

1. BARROS, G.C; SESSA, P.A; MATTOS, E. A. de; CARIAS, V. R.D; MAYRINK, W.; AIRES DE, J.T.; AIRES de; FALQUETO, A. \& JESUS, A.C. de - Foco de leishmaniose tegumentar americana nos municípios de Viana e Cariacica, Estado do Espírito Santo, Brasil. Rev. Saúde públ. (S.Paulo), 19: 146-153, 1985.

2. CONSOLIM, J.; LUZ, E. \& TORRES, P.B. - Aspectos biológicos dos flebotomíneos da área de Itaipu, no Estado do Paraná - Brasil - (Diptera Psychodidae). In: CONGRESSO BRASILEIRO DE ZOOLOGIA, 15, Curitiba 1988. p. 173. 
TEODORO, U., SPINOZA, R.P.; LA SALVIA FILHO, V.; GULLHERME, A.L.F.; LIMA, A.P.; JUNQUEIRA, G.M.B.; MISUTA, N.M.; NERILO SOBRINHO, A. \& LIMA, E.M. de - Da necessidade de se adotar e divulgar esquemas terapêuticos para tratamento de leishmaniose tegumentar no Paraná. Rev. Inst. Med. trop. S. Paulo, 33 (3): 199-204, 1991.

3. FORATTINI, O.P.; RABELLO, E.X.; SERRA, O.P.; COTRIM, M. das D.; GALATI, E.A.B. \& BARATA, J.M.S. - Observações sobre transmissão da leishmaniose tegumentar no Estado de São Paulo, Brasil. Rev. Saúde públ. (S.Paulo), 10:31-43, 1976.

4. FURTADO, T. \& VIEIRA, J.B.F. - Geografia da leishmaniose tegumentar americana no Brasil. An. bras. Derm., 57:135-140, 1982.

5. GOMES, A. de C. - Mecanismos e significados epidemiológicos da domiciliação. Rev. Saúde públ. (S.Paulo), 20:385-390, 1986.

6. GOMES, A de C.; RABELLO, E. X \& GALATI, E.A.B. Flebotomíneos encontrados em galinheiros nos Estados de São Paulo e Minas Gerais (Brasil) e algumas observações ecológicas. Rev. Saúde públ. (S. Paulo) 12:403-407, 1978.

7. GRIMALDI Jr., G.; DAVID, J.R. \& MCMAHONPRATT, D. - Identification and distribution of new world Leishmania species characterized by serodeme analysis using monoclonal antibodies. Amer. J. trop. Med. Hyg., 36:270-287, 1987.

8. LAINSON, R. - Our present knowledge of the ecology and control of leishmaniasis in the Amazon region of Brazil. Rev. Soc. bras. Med. trop., 18:47-56, 1985.

9. LIMA, L.C.R.; MARZOCHI, M.C. de A. \& SABROZA, P.C. - Flebotomíneos em áreas de ocorrência de leishmaniose tegumentar no bairro de Campo Grande, Rio de Janeiro, Brasil. Rev. bras. Malar., 33:64-74, 1981.

10. MAGALHÃES,. P.A. - Leishmaniose no Vale do Rio Doce. An. bras. Derm., 52:319-324, 1977.

11. MARSDEN, P.D. - Pentavalent antimonials: old drugs for new diseases. Rev. Soc. bras. Med., trop., 18:187-198, 1985.

12. MARSDEN, P.D. - Mucosal leishmaniasis ("espundia" Escomel, 1911). Trans. roy. Soc. trop. Med. Hyg, 80:859-876, 1986.

13. MINISTÉRIO DA SAÚDE - DN DS/SNPES DIENF/ DECEN/SUCAM. Relatório da Reunião Técnicooperacional sobre o controle da leishmaniose tegumentar americana (LTA), no Brasil, Brasinia 04-06/04/88.

14. RANGEL, E.F.; SOUZA, N.A. de; WERMELINGER, E.D. \& BARBOSA, A.F. - Infeç̧ão natural de Lu. intermedia Lutz \& Neiva, 1912, em área endêmica de leishmaniose tegumentar no Estado do Rio de Janeiro. Mem. Inst. Oswaldo Cruz. 79:395-396, 1984.

15. SALAZAR, H.C. \& SALGUEIRO, P.F. - Leishmaniasis epidemiological aspects in Brazil and in Rio de Janeiro. Rev. bras. Med., 36:594-597, 1979.

16. SAMPAIO, R.N.R.; SOARES, S.K.P.; ROSA, A. de C. MARTINS NETTO, E.; MAGALHÃES, A.V. \& MARSDEN, P.D. - Tratamento com pentamidina de seis casos da mucosa de leishmaniose tegumentar. An. bras. Derm., 63:439-442, 1988.
17. SILVEIRA, T.G.V.; TEODORO, U.; ARRAES, S.M.A.A.; LONARDONI, M.V.C.; DIAS, M.L.G.G.; SHAW, J.J.; ISHIKAWA, E. A. Y. \& LAINSON, R. - An autochthonous case of cutaneous leishmaniasis caused by Leishmania (Leishmania) amazonensis Lainson e Shaw, 1972 from the North of Parana State, Brazil. Mem. Inst. Oswaldo Cruz, 85:475-476, 1990.

18. SUCAM - DISTRITO DE LONDRINA - MINISTÉRIO DA SAÚDE. Relatório das atividades deste distrito face à existência de foco de leishmaniose $\mathrm{em}$ localidade dos municípios de: Terra Boa e Jussara - PR. Londrina, 11/08/78.

19. SUCAM - MINISTÉRIO DA SAÚDE. Leishmaniose tegumentar americana no Brasil: Sua problemática, seu controle, desafios e perspectivas. Inform. epidem. SUCAM, 5(50), 1987.

20. TALHARI, S.; SARDINHA, J.C.G.; SCHET'TINI, A.P.M.; ARIAS, J. \& NAIFF, R.D. - Tratamento de leishmanioses tegumentar americana. Resultados preliminares com pentamidina. An. bras. Derm. 60:361-364, 1985.

21. TALHARI, S.; ARIAS, J.R.; CUNHA, M.G.S.; NAIFF, R.D.; NAIFF, M.F.; FREITAS, R.A. de \& BARRETT, T. - Leishmaniose no Estado do Amazonas. Aspectos epidemiológicos, clínicos e terapêuticos. An. bras. Derm., 63:433-438, 1988.

22. TEODORO, U. - Aspectos epidemiológicos e do controle das leishmanioses americanas. São Paulo, 1987. (Dissertação de mestrado - Faculdade de Saúde Pública da Universidade de São Paulo).

23. TEODORO, U.; SPINOZA. R.P.; LA SALVIA FILHO. V.; GUILHERME, A.L.F.; LIMA, A.P. de; JUNQUEIRA, G.M.B.; NERILO SOBRINHO, A. \& LIMA, E.M. de Da necessidade de se adotar e divulgar esquema terapêutico para tratamento de leishmaniose tegumentar no Paraná. In: CONGRESSO DA SOCIEDADE BRASILEIRA DE MEDICINA TROPICAL, 20., Florianópolis, 1989. Resumos. Florianópolis, Imprensa Universitária da Universidade Federal de Santa Catarina, 1989. p. 57, re. $\pi^{\mathrm{o}}$ 149. (Rev. Soc. bras. Med. trop, 22(Supl.): 57, 1990).

24. VERZIGNASSI, T.G.; PEREIRA, D.S.; TEODORO, U.; MISUTA, N.M.; DIAS, M.L.G.G.; FERREIRA, M.E.M.C.; FRESSATTI, R. \& ARISTIDES, S.M.A. Leishmaniose tegumentar americana: aspectos epidemiológicos no Norte do Paraná - Brasil. In: REUNIÃO ANUAL DA SOCIEDADE BRASILEIRA PARA O PROGRESSO DA CIÊNCIA, 40., São Paulo, 1988. Resumos. São Paulo, Artes Gráficas Guaru, 1988. p.884-885. res. n 13-G.1.9. (Ciênc. e Cult., 40(Supl. 7): 884-885, 1988).

25. WARD, R.D. - New world leishmaniasis: a review of the epidemiological changes in the last three decades. In: INTERNATIONAL CONGRESS OF ENTOMOLOGY. 15., Washington, 1977. Proceedings. Washington, DC, 1977. p. 505-522. 